\title{
Efeito da adição de coating de cromita de ferro na emissividade de concreto refratário de alta alumina
}

\section{(Effect of iron chromite coating addition in the emissivity of a high alumina refractory castable)}

\author{
R. G. Campiteli ${ }^{1}$, M. R. Morelli ${ }^{2}$ \\ 'Programa de Pós-Graduação em Ciência e Engenharia de Materiais, \\ ${ }^{2}$ Departamento de Engenharia de Materiais, Universidade Federal de S. Carlos, \\ Rod. Washington Luis, km 235, S. Carlos, SP, Brasil \\ rgcampiteli@gmail.com,morelli@ufscar.br
}

\begin{abstract}
Resumo
Coatings de alta emissividade têm sido utilizados em aplicações industriais há mais de 40 anos com o objetivo de reduzir as perdas térmicas em processos de aquecimento. Com a aplicação de um coating de alta emissividade na superfície dos revestimentos internos de um forno industrial é possível aumentar a eficiência nas trocas térmicas entre a atmosfera e as paredes do revestimento, reduzindo a perda de calor e também o consumo de combustíveis. Em geral são utilizados compostos cerâmicos como óxido de cério, carbeto de boro, boreto de silício, siliceto de molibidênio ou óxido de cromo como agentes de emissividade para se obter as propriedades termo-ópticas desejadas nos coatings. Entretanto tais compostos muitas vezes inviabilizam sua aplicação, devido ao seu elevado valor comercial ou ainda sua escassez. Neste contexto, o presente trabalho buscou avaliar os efeitos da cromita de ferro, um óxido mineral abundante e de valor comercial acessível, em sua utilização como agente de emissividade em coatings refratários de alta emissividade. Por meio de um método indireto de medição de emissividade, foram avaliadas de maneira comparativa composições com e sem a presença da cromita de ferro visando sua aplicação como cobertura de revestimentos isolantes e refratários de fornos de aquecimento industrial. Com adições de 7,5\% de cromita de ferro, observaram-se aumentos na emissividade dos coatings da ordem de $8 \%$, sugerindo seu potencial para essa aplicação.
\end{abstract}

Palavras-chave: tinta de alta emissividade, cromita de ferro.

\begin{abstract}
High emissivity coatings have been used in industrial applications over 40 years with the goal of reducing the thermal losses in heating processes. By applying a high emissivity coating on the internal surface of a kiln lining, it is possible to increase the efficiency in heat exchange between the atmosphere and the walls, reducing the heat loss and also the fuel consumption. In general ceramic compounds such as cerium oxide, boron carbide, silicon boride, molybdenum silicide or chromium oxide are used as emissivity agents to obtain the desired thermo-optical properties in coatings. However, such compounds often prevent their application due to its high commercial value or its scarcity. In this context, this study aimed to evaluate the effects of iron chromite, an abundant mineral oxide and affordable commercial value in its use as emissivity agent in high emissivity refractory coatings. Through an indirect method of measuring emissivity, different coatings compositions with and without the addition of iron chromite were evaluated, in a comparative manner, aiming its application as coatings for refractory and insulation linings of industrial heating furnaces. With iron chromite additions of 7.5\%, there was almost $8 \%$ increase in the emissivity of the coatings, suggesting their potential for this application.
\end{abstract}

Keywords: high emissivity coatings, iron chromite.

\section{INTRODUÇÃO}

O consumo de energia em processos industriais de aquecimento é tema recorrente nas indústrias e organizações de todo o mundo, tanto na questão econômica quanto na questão ambiental. Esforços são realizados continuamente para se reduzir o uso de combustíveis ou outras fontes de energia nesses processos, por meio da otimização e aumento da eficiência em fornos de secagem, aquecimento, incineração, calcinação, tratamento ou fusão de materiais em altas temperaturas. Uma das tecnologias que permite um melhor aproveitamento da energia gerada para o aquecimento de fornos industriais é o uso de coatings de alta emissividade. São composições cerâmicas, desenvolvidas para aumentar a emissividade superficial dos revestimentos refratários, utilizados como isolamentos térmicos de equipamentos que operam em temperaturas elevadas, e consequentemente para reduzir a dissipação de calor para o exterior. Relatos na literatura sugerem que é possível se obter uma redução de até $6,2 \%$ no consumo energético de fornos industriais com a aplicação de uma camada superficial de alta emissividade sobre os revestimentos internos desses equipamentos [1]. 
Transferência de calor e emissividade: sempre que houver uma diferença de temperatura entre as regiões de um meio, haverá transferência de calor da região de maior temperatura para a de menor temperatura [2]. Um dos mecanismos de transferência de calor de maior relevância para os processos de aquecimento industrial é a radiação térmica. A radiação térmica é definida como a energia radiante emitida por um meio devido à sua temperatura e difere dos outros mecanismos de transferência de calor, pois não necessita de um suporte material para ocorrer, isto é, mesmo no vácuo é possível se emitir energia radiante. Qualquer corpo cuja temperatura é superior a zero absoluto, está constantemente emitindo radiação térmica por ondas eletromagnéticas em comprimentos de onda de 0,1 a 100 $\mu \mathrm{m}$ [3]. O fluxo de calor máximo emitido por um corpo por radiação térmica $\left(Q_{\text {emit.máx }}\right)$ pode ser representado pela equação de Stefan Boltzmann, dada por:

$$
\mathrm{Q}_{\text {(emit.máx.) }}=\sigma \cdot \mathrm{A}_{\mathrm{s}} \cdot \mathrm{T}_{\mathrm{s}}^{4}
$$

onde, $\sigma$ é a constante de Stefan Boltzmann $\left[5,67 \times 10^{-8} \mathrm{~W} /\left(\mathrm{m}^{2} \mathrm{~K}^{4}\right)\right]$, $A_{s}$ é a área da superfície do corpo e $T_{s}$ é a sua temperatura superficial. A Equação A descreve a taxa máxima de calor emitida por um corpo idealizado, conhecido como corpo negro [3]. Nesse material ideal, toda a radiação recebida é absorvida e então reemitida. Já em superfícies reais, apenas uma fração da energia recebida é absorvida e a taxa de radiação emitida é sempre menor que a radiação de um corpo negro. $O$ fator que define essa proporção de energia emitida é conhecido como emissividade. Portanto em corpos reais, a equação de Stefan Boltzmann pode ser redefinida para:

$$
\mathrm{Q}_{\text {(emit.máx.) }}=\varepsilon . \sigma \cdot \text { As. }_{\mathrm{s}}^{4}
$$

onde, $\varepsilon$ é a emissividade do corpo. A emissividade de um material pode ser definida como sua habilidade em absorver e irradiar calor: varia sempre entre 0 e 1 e é adimensional. Uma emissividade igual a 0 caracteriza um refletor perfeito, ou seja, toda a energia que atinge essa superfície é refletida. Já uma emissividade igual a 1 remete a um corpo negro, onde toda a energia incidente é absorvida. A emissividade de uma superfície pode variar de acordo com sua rugosidade, temperatura, a direção e o comprimento de onda incidente.

De acordo com o princípio de conservação de energia, a energia incidente em uma superfície por meio de ondas eletromagnéticas pode interagir com o meio de três maneiras [4]: ela pode ser absorvida (a), refletida (r) ou transmitida ( $t$ ), e a soma das frações de cada processo de interação deve sempre ser igual a 1, de acordo com a Equação C. Dessa maneira, no interior de um forno industrial, a energia radiante que atinge as suas paredes internas pode ser refletida novamente para a atmosfera do equipamento ou absorvida pelo revestimento, uma vez que a transmissão de ondas eletromagnéticas só ocorre em materiais semitransparentes ou transparentes. Entretanto, é importante ressaltar que a energia emitida pelos gases aquecidos da atmosfera de um forno compreende uma limitada faixa de comprimentos de onda. Quando refletida pela parede, a energia retorna ao interior do equipamento com a mesma faixa de comprimentos de onda incidente. Porém, se essa energia for absorvida pela superfície da parede e então irradiada novamente, ela compreenderá uma nova faixa de comprimentos de onda, que não é absorvida pelos gases e sim pela carga em aquecimento [5].

$$
\mathrm{a}+\mathrm{r}+\mathrm{t}=1
$$

Outro aspecto que deve ser considerado, para que haja eficiência do coating de alta emissividade em fornos, é que deve haver uma diferença de temperatura do interior do forno e a parede interna, sendo que esta última deve ser maior [6], para que o fluxo de calor dado pela Equação D seja favorável. Conforme a Lei de Kirchoff, em equilíbrio termodinâmico, para uma mesma temperatura e comprimento de onda, em uma dada superfície a emissividade é igual à absortividade $(\varepsilon=\alpha)$, ou seja, toda energia absorvida é emitida. Com isso, pode-se obter que a taxa líquida de calor irradiado $\left(\mathrm{Q}_{\mathrm{rad}}\right)$ pela parede para a carga é diretamente proporcional à diferença de temperatura entre essas regiões, de acordo com a Equação D:

$$
\mathrm{Q}_{\text {(rad.) }}=\varepsilon . \sigma . A s\left(\mathrm{~T}_{\mathrm{s}}^{4}-\mathrm{T}_{\mathrm{i}}^{4}\right)
$$

onde, $\mathrm{T}_{\mathrm{s}}$ é a temperatura da superfície da parede e $\mathrm{T}_{\mathrm{i}}$ é a temperatura do interior do forno. Quando houver equilíbrio térmico entre a parede e o interior do forno, não haverá mais fluxo de calor por radiação e a taxa líquida de calor será zero. Isso ocorre, por exemplo, em patamares de manutenção de temperatura durante um ciclo de aquecimento e nessa condição os coatings de alta emissividade não apresentam qualquer benefício. Por fim, durante o resfriamento dos fornos, quando as fontes de aquecimento são desligadas, as paredes perdem calor mais rapidamente que o interior e a carga, portanto a taxa líquida de calor será negativa. Isso significa que o coating de alta emissividade aumenta a absorção de calor da superfície, cuja maior parte agora será conduzida através do revestimento para o exterior do forno. Ou seja, o benefício do aumento da emissividade superficial do revestimento interno ocorre somente durante o aquecimento ou o resfriamento, tornando essas etapas mais rápidas e eficientes. Pode-se obter um aumento na velocidade do resfriamento de $13,5 \%$ com o uso de coatings de alta emissividade em revestimento de tijolos refratários de sílica [7].

Agentes de emissividade: de maneira geral, a emissividade dos materiais refratários utilizados no revestimento isolante de fornos industriais diminui com o aumento da temperatura. Normalmente esses revestimentos apresentam emissividades da ordem de 0,3 na temperatura de operação do equipamento, o que significa que apenas $30 \%$ da energia radiante incidente na superfície do revestimento é absorvida e reemitida para o interior do forno. O uso de um coating de alta emissividade pode aumentar esse percentual para 85 a 95\% [8]. Para que um coating de alta emissividade mantenha suas propriedades termo-ópticas mesmo com o 
aumento da temperatura, é necessário o uso de agentes de emissividade, ou seja, compostos cerâmicos que apresentam elevadas emissividades na temperatura de operação dos fornos industriais, tais como $\mathrm{SiC}, \mathrm{Cr}_{2} \mathrm{O}_{3}, \mathrm{Fe}, \mathrm{B}_{4} \mathrm{C}, \mathrm{BN}, \mathrm{AlN}$, $\mathrm{ZrO}_{2}, \mathrm{TiO}_{2}, \mathrm{Fe}_{2} \mathrm{O}_{3}, \mathrm{MgO}, \mathrm{CeO}_{2}, \mathrm{~Tb}_{2} \mathrm{O}_{3}, \mathrm{FeTiO}_{3}, \mathrm{SiB}_{6}, \mathrm{SiB}_{4}$, $\mathrm{MoSi}_{2}, \mathrm{WSi}_{2}, \mathrm{ZrB}_{2}, \mathrm{Si}_{3} \mathrm{~N}_{4}, \mathrm{Co}_{3} \mathrm{O}_{4}, \mathrm{NiO}, \mathrm{Cu}_{2} \mathrm{Cr}_{2} \mathrm{O}_{5}$ e $\mathrm{ZrSiO}_{4}$ [1, 6-11]. A maioria dos compostos citados é semicondutora que necessita de menor quantidade de energia para promover seus elétrons da banda de valência para a de condução.

Cromita de ferro: os agentes de emissividade normalmente citados na literatura são em geral escassos e muitas vezes os elevados custos inibem sua utilização em coatings de alta emissividade. A cromita de ferro trata-se de um mineral abundante utilizado como principal fonte de obtenção de óxido de cromo. É largamente utilizada em aplicações refratárias, devido ao seu alto ponto de fusão $(2180$ ${ }^{\circ} \mathrm{C}$ ), expansão térmica reversível moderada (cerca de 1,3\% a $1400^{\circ} \mathrm{C}$ ), neutralidade química frente ao contato com metais líquidos e resistência à corrosão relativamente elevada $[12$, 13]. Por se tratar de um óxido complexo, majoritariamente composto por $\mathrm{FeO} . \mathrm{Cr}_{2} \mathrm{O}_{3}$, além da presença significativa de $\mathrm{MgO}$ e $\mathrm{Al}_{2} \mathrm{O}_{3}$ em sua composição, apresenta características do ponto de vista físico-químico e comercial potenciais para sua utilização em coatings de alta emissividade. Nenhuma referência bibliográfica foi encontrada, até o momento, descrevendo o uso da cromita de ferro como agente de emissividade em coatings de alta emissividade. Neste contexto, o presente trabalho buscou avaliar os efeitos da cromita de ferro como agente de emissividade em coatings refratários de alta emissividade.

\section{EXPERIMENTAL}

Neste trabalho foi formulado um coating de alta emissividade, com pós cerâmicos e aditivos listados na Tabela I. Para prepará-lo foi utilizado um agitador mecânico de elevada rotação (até $5000 \mathrm{rpm}$ ), com tempo de mistura de $10 \mathrm{~min}$ a aproximadamente $2000 \mathrm{rpm}$. Em seguida o coating foi aplicado sobre um corpo de prova fabricado em concreto refratário de aproximadamente $70 \%$ de alumina, previamente sinterizado, com o auxílio de uma pistola de ar comprimido. A espessura de aplicação foi definida em 200 $\pm 20 \mu \mathrm{m}$, controlada por desbaste realizado com uma lixa de gramatura 320 e auxílio de um medidor de profundidade Digimess com precisão de $10 \mu \mathrm{m}$. Depois de aplicados, os coatings foram secos ao ar por $24 \mathrm{~h}$ e então em estufa com temperatura controlada a $110{ }^{\circ} \mathrm{C}$ por mais $24 \mathrm{~h}$. Para efeito de comparação da eficiência da cromita como agente de emissividade, foi também formulada uma composição sem a presença de qualquer agente de emissividade, sendo a quantidade destinada a esse componente, adicionada à carga refratária.

Os corpos de prova foram submetidos a ensaios em um dispositivo construído para esse trabalho, onde foi realizada a medição indireta da emissividade. $\mathrm{O}$ dispositivo consiste em uma placa de aquecimento elétrico, com cavidades próprias para o alojamento dos corpos de prova, com termopares de referência e um pirômetro infravermelho com ajuste de emissividade de 0,10 a 0,99 da Instrutherm para até $1600{ }^{\circ} \mathrm{C}$. A Fig. 1 ilustra de maneira esquemática a disposição e funcionamento do dispositivo. Para realização das medições indiretas de emissividade, um corpo de prova sem a aplicação de qualquer coating foi posicionado em uma das duas cavidades, onde foi acoplado um termopar de referência. Na outra cavidade foi posicionado um corpo de prova com o coating em análise. A temperatura da placa de aquecimento foi controlada em um controlador da Alutal, FAY $500 \mathrm{~A}$, com precisão de $1{ }^{\circ} \mathrm{C}$. Foram realizados ensaios nas temperaturas de 800,900 e $1000^{\circ} \mathrm{C}$, sendo a temperatura de $1000{ }^{\circ} \mathrm{C}$ a temperatura limite do equipamento. Os resultados foram obtidos pela média de 3 medições para cada corpo de prova. Em frente à placa de aquecimento $\mathrm{e}$ aos corpos de prova, foi posicionado o pirômetro, preso a um trilho de deslocamento lateral, visando permitir seu deslocamento para medição em ambos os corpos de prova, porém garantindo sua perpendicularidade a eles.

Foram programadas as temperaturas no controlador de aquecimento, até se obter no termopar de referência o valor de temperatura desejado para a realização dos ensaios. Ao atingir essa temperatura, aguardou-se $10 \mathrm{~min}$ para que o equilíbrio térmico fosse atingido entre o corpo de prova e a placa de aquecimento. O pirômetro foi posicionado em frente ao corpo de prova e então sua emissividade indicada foi ajustada até que a temperatura indicada em seu mostrador se igualasse à temperatura do termopar de referência, permitindo-se uma tolerância de $10{ }^{\circ} \mathrm{C}$ para mais ou para menos. Após esse ajuste, foi tomada a emissividade ajustada como a emissividade da superfície sob medição. Vale ressaltar que essa medição indireta é apenas qualitativa para comparação entre as composições dos coatings e a

Tabela I - Quantidades em massa dos componentes da composição do coating e origem dos materiais utilizados. [Table I - Quantities by weight of components of coating composition and the origin of used materials.]

\begin{tabular}{lcl}
\hline Componente & $\%$ & Origem \\
\hline Sílica eletrofundida \#325 mesh (carga refratária) & 46,0 & Imerys Fused Minerals Salto Ltda \\
Veículo serigráfico 1002 PG & 20,0 & PG Química \\
Bentonita sódica natural (aditivo espessante) & 1,5 & Base Química \\
Sílica coloidal 1440 (aditivo ligante) & 25,0 & Eka Chemicals \\
Cromita \#325 mesh (agente de emissividade) & 7,5 & Oxidry Minerals Ltda \\
\hline
\end{tabular}




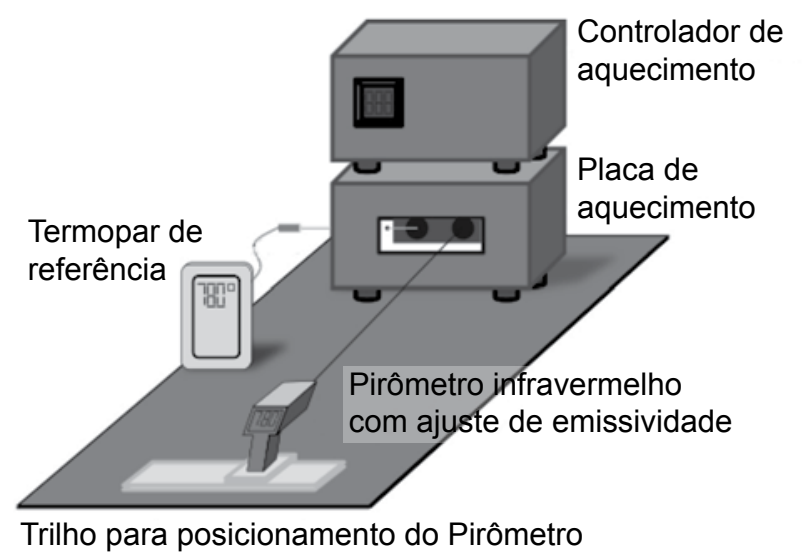

Figura 1: Ilustração esquemática do dispositivo construído para medição indireta de emissividade.

[Figure 1: Schematic illustration of the device built for indirect measurement of emissivity.]

superfície do corpo de prova sem recobrimento, não devendo ser considerada como a emissividade real das superfícies. Por fim, foi realizada uma análise morfológica nos corpos de prova recobertos por meio de microscopia eletrônica de varredura (MEV) em equipamento Inspect 550, operado a 25 $\mathrm{kV}$. Foi realizada também a microanálise por espectroscopia de energia dispersiva (EDS), com o auxílio do software EDAX Genesis.

\section{RESULTADOS E DISCUSSÃO}

\section{Medição indireta de emissividade}

Os resultados obtidos pela medição indireta da emissividade, através do dispositivo construído nesse trabalho, apontaram apenas um valor qualitativo da emissividade, permitindo a comparação entre a composição com e sem adição de cromita. Portanto, os valores de emissividade obtidos não representam o valor real de emissividade dessas superfícies. Partindo da Equação $\mathrm{D}$, onde as temperaturas são elevadas à quarta potência, e considerando que os valores de $\mathrm{T}_{\mathrm{s}}$ (temperatura da superfície dos corpos de prova) foram 800,900 e $1000{ }^{\circ} \mathrm{C}$, $\mathrm{T}_{\mathrm{i}}$ (temperatura ambiente, externa ao dispositivo) torna-se desprezível. Sendo assim, o calor de radiação vindo da placa de aquecimento pode ser dado de maneira simplificada por:

$$
\mathrm{Q}_{\text {(rad.) }}=\varepsilon \cdot \sigma \cdot \mathrm{A}_{\mathrm{s}} \cdot \mathrm{T}_{\mathrm{s}}^{4}
$$

Como o calor de radiação emitido pela placa de aquecimento é constante, devido à presença das resistências elétricas trabalhando para manter a temperatura estável, pode-se considerar que:

$$
\mathrm{Q}_{\text {(rad.) }}=\text { Constante }=\varepsilon \cdot \sigma \cdot \mathrm{A}_{\mathrm{s}} \cdot \mathrm{T}_{\mathrm{s}}^{4}
$$

Como o dispositivo construído para medição indireta da emissividade utiliza como referência a temperatura, equiparando-a com o termopar acoplado ao corpo de prova através do ajuste da emissividade no pirômetro, pode-se concluir que com um aumento da emissividade superficial dos corpos de prova (ao introduzir um coating) deve ser observado na verdade por uma redução da emissividade medida pelo pirômetro, uma vez que $\mathrm{T}_{\mathrm{s}}$ é fixo, pois como mostrado na Equação $\mathrm{F}$ o produto entre $\varepsilon$ e $\mathrm{T}_{\mathrm{s}}{ }^{4}$ deve-se manter constante. Na Fig. 2 são apresentados os resultados da medição indireta de emissividade para 3 diferentes situações: um corpo de prova sem aplicação de coating, simulando o revestimento refratário comum de um forno, um corpo de prova com a aplicação de um coating sem adição do agente de emissividade e por fim um corpo de prova com a aplicação de um coating com adição de cromita, composição apresentada na Tabela I.

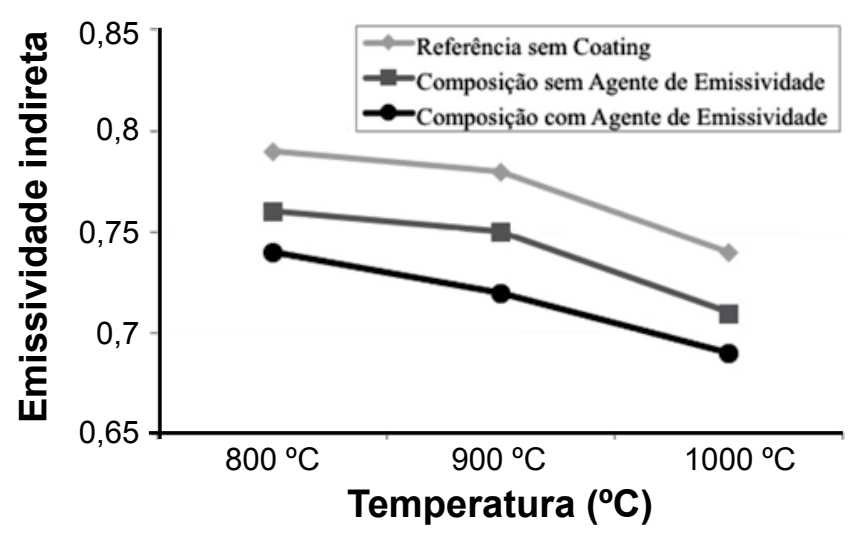

Figura 2: Resultados de medição indireta de emissividade em função da temperatura para um corpo de prova sem qualquer aplicação de coating, a composição sem qualquer adição de um agente de emissividade e a composição com adição de um agente de emissividade (cromita).

[Figure 2: Results of the indirect emissivity measurement as a function of temperature for a sample without any coating applied, a composition without any emissivity agent added and a composition with an addition of an emissivity agent (chromite).]

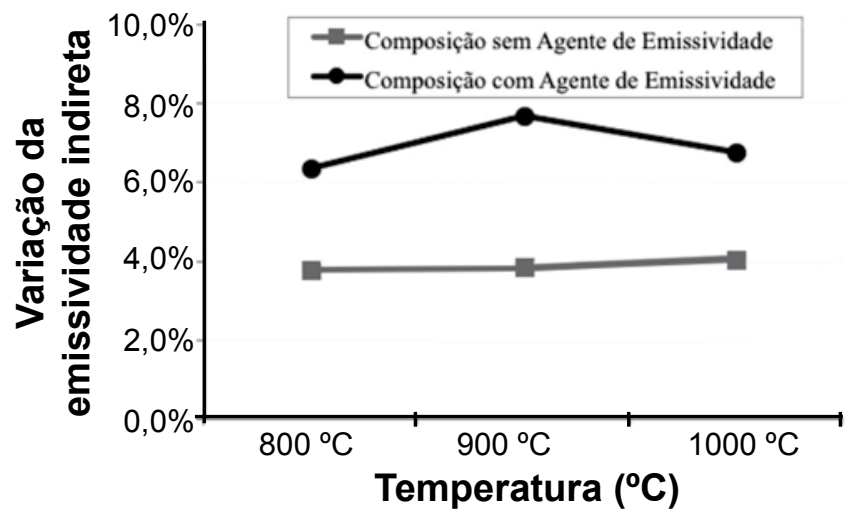

Figura 3: Gráfico da variação da emissividade indireta em função da temperatura das composições com e sem adição do agente de emissividade em relação ao corpo de prova sem coating.

[Figure 3: Graphic of the indirect emissivity variation as a function of temperature for the coatings compositions with and without addition of an emissivity agent in relation to the sample without any coating.] 
O comportamento de diminuição no valor da emissividade mostrado na Fig. 2 com o aumento da temperatura e com as adições dos coatings, conforme explicado anteriormente, é coerente ao considerar que uma maior emissividade representa uma maior eficiência de emissão de radiação. Uma vez que o calor absorvido pelo corpo de prova é emitido para o ambiente de maneira mais eficiente, devese esperar uma redução em sua temperatura superficial, porém como a temperatura foi o parâmetro de referência do ensaio (regulando-se a emissividade do pirômetro, de modo a manter a temperatura mostrada igual à do termopar de referência) e o produto entre $\varepsilon$ e $\mathrm{T}_{\mathrm{s}}^{4}$ deve-se manter constante, observa-se então uma redução na emissividade. Tal fenômeno já havia sido observado na literatura [14]. Com base nesse conceito da medição indireta, é possível
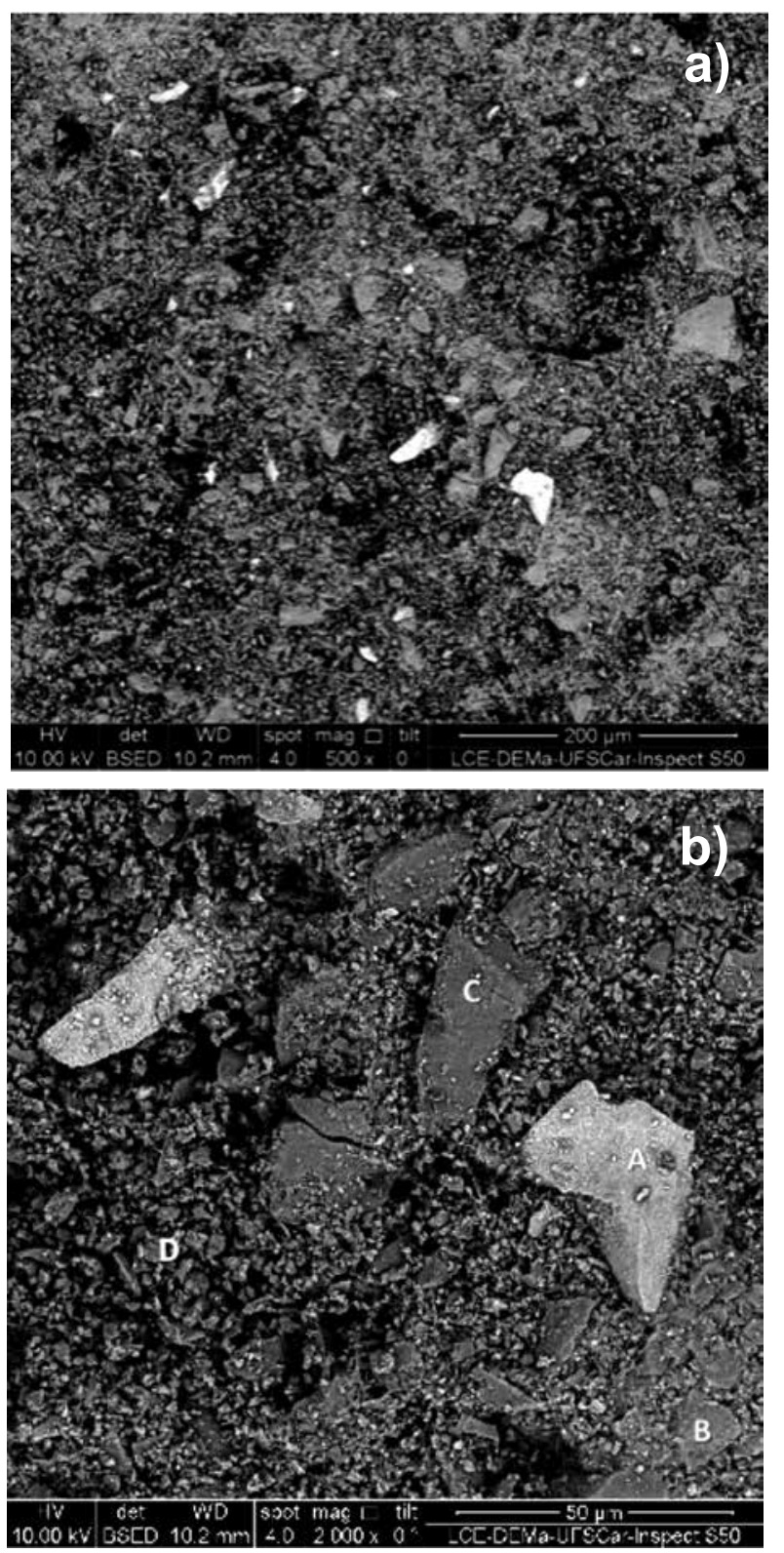

Figura 4: Micrografias obtidas por microscopia eletrônica de varredura do coating de alta emissividade.

[Figure 4: SEM micrographs of the high emissivity coating.] avaliar que houve de fato um aumento da emissividade com a adição dos coatings, para todas as temperaturas, em relação ao corpo de prova de referência sem o recobrimento. Nota-se ainda que dentre os corpos de prova com a pintura a adição da cromita resultou em uma elevação dos valores de emissividade. Para melhor ilustrar esse ganho nas propriedades termo-ópticas com a aplicação dos coatings de alta emissividade, a Fig. 3 representa a variação obtida na emissividade em relação ao corpo de prova isento de pintura para cada temperatura de ensaio. Nessa figura é possível observar claramente o ganho de emissividade em cada composição. Com a adição da cromita, nota-se ganhos de 6 a $8 \%$ na emissividade indireta, enquanto o coating sem um agente de emissividade apresentou ganhos da ordem de $4 \%$ em todas as temperaturas.

\section{Microscopia eletrônica de varredura}

A observação da morfologia do coating formulado conforme Tabela I, através de uma análise de MEV mostrou que o agente de emissividade foi bem disperso na matriz de sílica eletrofundida e mesmo após a realização do ensaio de medição indireta de emissividade, no qual o material foi submetido a temperaturas de até $1000{ }^{\circ} \mathrm{C}$,

Tabela II - Composição química obtida por microanálise EDS nos pontos A, B, C e D apontados na Fig. 4b.

[Table II - Chemical composition obtained by EDX microanalysis in the points $A, B, C$ and D shown in Fig. 4b.]

\begin{tabular}{cccccccc}
\hline Ponto & $\begin{array}{c}\mathrm{Mg} \\
(\%)\end{array}$ & $\begin{array}{c}\mathrm{Al} \\
(\%)\end{array}$ & $\begin{array}{c}\mathrm{Si} \\
(\%)\end{array}$ & $\begin{array}{c}\mathrm{Ti} \\
(\%)\end{array}$ & $\begin{array}{c}\mathrm{Cr} \\
(\%)\end{array}$ & $\begin{array}{c}\mathrm{Fe} \\
(\%)\end{array}$ & $\begin{array}{c}\mathrm{O} \\
(\%)\end{array}$ \\
\hline $\mathrm{A}$ & 13,15 & 17,56 & 1,80 & 0,12 & 7,81 & 2,41 & 57,15 \\
$\mathrm{~B}$ & 0,24 & 0,77 & 45,30 & 0,04 & 0,05 & 0,04 & 53,57 \\
$\mathrm{C}$ & 0,28 & 0,65 & 43,19 & 0,06 & 0,09 & 0,07 & 55,67 \\
$\mathrm{D}$ & 0,84 & 1,46 & 42,64 & 0,09 & 0,36 & 0,21 & 54,51 \\
\hline
\end{tabular}

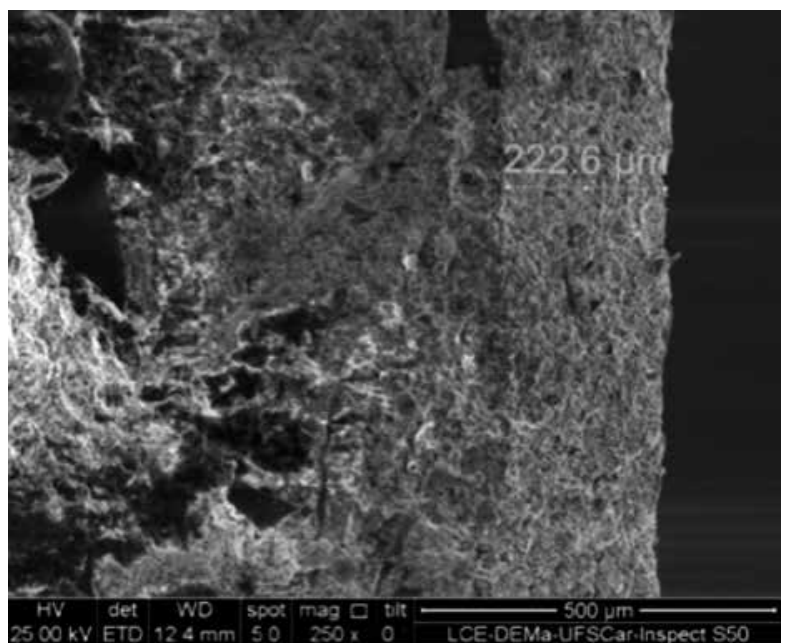

Figura 5: Micrografia obtida por microscopia eletrônica de varredura da interface entre o coating e seu substrato.

[Figure 5: SEM micrograph of the interface between the coating and its substrate.] 
sua microestrutura permaneceu estável, sem a presença de defeitos microestruturais. Os grãos mais claros foram identificados como a cromita através da microanálise pontual. As Figs. 4 e 5, respectivamente, apresentam as micrografias obtidas do coating e da interface entre o coating e o substrato. A Fig. $4 \mathrm{~b}$ indica os pontos onde foram realizadas as microanálises por EDS e a Tabela II seus resultados, onde claramente o ponto A pode ser identificado como cromita e os pontos B, C e D como a sílica eletrofundida utilizada como carga. Na Fig. 5, pode ser observado que houve boa aderência do coating ao seu substrato ao não evidenciar falhas entre as duas microestruturas.

\section{CONCLUSÕES}

A seleção de compostos cerâmicos para uso em coatings de alta emissividade consistiu na análise de materiais viáveis economicamente para substituir os agentes citados na literatura que, devido ao seu alto custo e escassez industrial, muitas vezes inviabilizam sua utilização. Esse trabalho teve como principal objetivo desenvolver uma metodologia e com ela realizar a medição indireta da emissividade da cromita de ferro, visando sua utilização como agente de emissividade em coatings de alta emissividade. $\mathrm{O}$ método proposto de medição indireta de emissividade mostrou-se capaz de comparar diferentes coatings, ainda que não permita uma medição quantitativa dessa propriedade. Observou-se que a temperatura da superfície de um coating com a presença de um agente de emissividade é menor em relação ao mesmo coating sem qualquer agente. Foi possível verificar através do ensaio de medição indireta de emissividade que a adição de cromita de ferro como agente de emissividade apresentou ganhos de emissividade da ordem de 6 a $8 \%$ em relação a um corpo de prova sem qualquer recobrimento. A composição manteve-se estável, sem apresentar falhas microestruturais mesmo após ser submetida a temperaturas de até $1000{ }^{\circ} \mathrm{C}$.

\section{REFERÊNCIAS}

[1] I. Benko, Periodica Polytechnica Ser. Mech. Eng. 35, 4 (1991) 235-245.

[2] F. Kreith, R.M. Manglik, M.S. Bohn, Principles of heat transfer, $7^{\mathrm{a}}$ Ed., Cengage Learning, Stamford, EUA (2011) 3.

[3] Y.A. Çengel, A.J. Ghajar, Transferência de calor e massa: uma abordagem prática, $4^{\mathrm{a}}$ Ed., Mcgraw Hill, Porto Alegre, Brasil (2012) 28.

[4] A.A.C. Mourão, D.O. Vivaldini, V.R. Salvini, V.C. Pandolfelli, Cerâmica 61, 357 (2015) 41-51.

[5] J.C. Hellander, Hydrocarbon Process. 76, 11 (1997) 9195.

[6] P.C. Sheil, T.R. Kleeb, AISTech - Iron and Steel Technology Conference Proceedings, Charlotte, EUA, Vol. 1 (2005) 527.

[7] Z. Dan, D. Cang, H. Zhou, H. Bai, Y. Zong, J. University Sci. Technology Beijing 15, 5 (2008) 627-632.

[8] D.V. Chauhan, S.N. Misra, R.N. Shukla, Der Chemica Sinica 3, 3 (2012) 621-627.

[9] M. Mauer, P. Kalenda, M. Honner, P. Vacikova, J. Phys. Chem. Solids 73 (2012) 1550-1555.

[10] X. He, Y. Li, L. Wang, Y. Sun, S. Zhang, Thin Solid Films 517, 17 (2009) 5120-5129.

[11] T.G. Evans, J. W. Olver, J.G. Dillard, J.A. Simmons, R.A. Churchward, "Thermal protective coating for ceramic surfaces", US Patent 6921431 (2005).

[12] N. McEwan, T. Courtney, R.A. Parry, P. Knupfer, Southern African Pyrometallurgy, Ed. R.T. Jones, P. den Hoed, Southern African Institute of Mining and Metallurgy, Johannesburg (2011) 359-371.

[13] W.W.K.K. Oo, S.W.H. Aye, K.T. Lwin, Int. J. Chem. Mol. Nucl. Mater. Metall. Eng. 2, 10 (2008) 234-239.

[14] R.L. Sala, M.A.L. Braulio, B.H. Teider, J.B. Gallo, V.C. Pandolfelli, "Aplicação de tintas de alta emissividade na indústria do alumínio", V Congresso Internacional do Alumínio, S. Paulo, Brasil (2012).

(Rec. 15/11/2015, Rev. 10/02/2016, 24/02/2016, Ac. 24/02/2016) 